\title{
Single-cell analysis for drug development using convex lens-induced confinement imaging
}

\author{
Ndeye Khady Thiombane', Nicolas Coutin', Daniel Berard², Radin Tahvildari ${ }^{2}$, Sabrina Leslie ${ }^{2}$ \& Corey Nislow $^{\star, 1}$
}

\section{GRAPHICAL ABSTRACT}

\section{Convex lens-induced confinement (CLiC)}

Extended high-throughput single-cell analysis of growth, proliferation and drug response

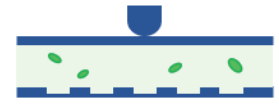

Flow cells contain micronscale pits to trap cells

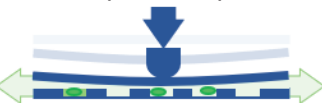

Cells squeezed into pits. Excess media expelled, improving SNR

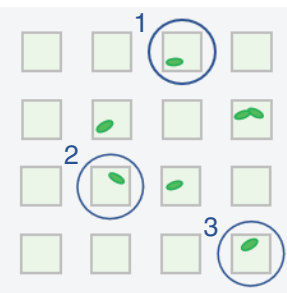

Cells confined to pits for extended observation
1

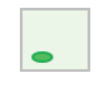

2

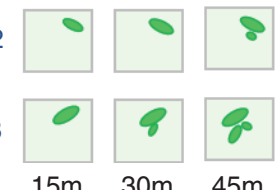

$15 \mathrm{~m} 30 \mathrm{~m}$

Single-cell measurements
reveal heterogeneity
Coverslips

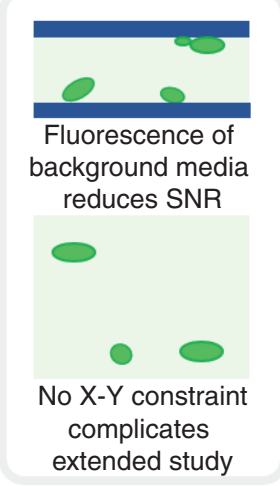

\section{ABSTRACT}

New technologies have powered rapid advances in cellular imaging, genomics and phenotypic analysis in life sciences. However, most of these methods operate at sample population levels and provide statistical averages of aggregated data that fail to capture single-cell heterogeneity, complicating drug discovery and development. Here we demonstrate a new single-cell approach based on convex lens-induced confinement (CLiC) microscopy. We validated CLiC on yeast cells, demonstrating subcellular localization with an enhanced signal-to-noise and fluorescent signal detection sensitivity compared with traditional imaging. In the live-cell CLiC assay, cellular proliferation times were consistent with flask culture. Using methotrexate, we provide drug response data showing a fivefold cell size increase following drug exposure. Taken together, CLiC enables highquality imaging of single-cell drug response and proliferation for extended observation periods.

\section{KEYWORDS}

$\mathrm{CLiC} \cdot$ dose-response $\cdot$ methotrexate $\cdot$ phenotypic screen $\cdot$ single cell $\cdot$ yeast

'Pharmaceutical Sciences, 2405 Wesbrook Mall, University of British Columbia, Vancouver, BC, V6T1Z3, Canada; ${ }^{2}$ Department of Physics, 214 Rutherford Physics Building, Mc Gill University, 3600 rue University, Montreal, QC, H3A 2T8, Canada; *Author for correspondence: corey.nislow@ubc.ca

BioTechniques 67: 210-217 (November 2019) 10.2144/ btn-2019-0067

\section{METHOD SUMMARY}

In this study, we use convex lens-induced confinement (CLiC) microscopy, which was originally developed for the study of single particles and biomolecules, and apply it to live cell imaging to detect fluorescently labeled cell compartments and compare the system to conventional imaging. We also performed an extended duration live-cell assay in CLiC by confining Saccharomyces cerevisiae yeast cells in well-defined pits in a flow cell and observing single-cell proliferation for $8 \mathrm{~h}$. Finally, we assessed the effects of the antifolate drug methotrexate on cell morphology and mitochondria integrity by observing cells during drug exposure.

One of the driving forces in systems biology research is the emergence and development of new data-acquisition tools and technologies to better understand biological and biochemical mechanisms at the single-cell level. At present, most practical molecular and cell biology methods capture the average response of a cell sample population. However, results from these approaches can be misleading due to cellular heterogeneity. Such variation may arise from diverse genetic and nongenetic factors, including noise in gene expression. Factors that substantially define the amplitude of such noise include regulatory dynamics, transcription rates, genetic factors and intracellular copy numbers of molecules involved in the genetic network [1].
Single-cell heterogeneity is indeed a well-known phenomenon in microbial resistance and evolution studies, as well as in cancer research. For instance, most cancer cell lines display genomic alterations, as well as other forms of heterogeneity such as chromosomal instability [1,2]. Stochastic cellular process fluctuations, including the state, location, activity and concentration of polymerases, transcription factors and gene expression regulators, may also lead to diversification in an initially uniform cell population [3]. These variations can have a significant impact on the growth and environmental stress response of cells and cannot be captured using conventional cell biology methods. For instance, conducting a drug response analysis in yeast often requires recording its 
optical density $\left(\mathrm{OD}_{600 \mathrm{~nm}}\right)$ at set time intervals over time. However, the recorded data are a bulk representation of the entire population and, as a consequence, may overlook fluctuations at the individual cell level that could lead to drug resistance [4].

The awareness of cell population heterogeneity and the importance of characterizing it have led to the emergence of a number of single-cell analysis tools such as fluorescence-activated cell sorting, microfluidic lab-on-a-chip and cellular microarrays. Microfluidics are a powerful, relatively recent innovation that are being applied for rapid antibiotic and antifungal susceptibility testing at a single-cell level, which can allow one to determine an isolate's drug susceptibility in less than $30 \mathrm{~min}[5,6]$. A range of antibiotic and antifungal drugs (e.g., penicillins such as mecillinam, amoxicillin-clavulate ampicillin, sulfamethoxazole, and so on) have been investigated using microfluidic lab-on-a-chip, and the results emphasize the utility of this technique to identify individual cells that maintain their fast growth rate against a background of drug susceptible cells [5]. The impact of single-cell analysis techniques on drug discovery and drug resistance studies in recent years underscores the clear need for more techniques that are robust in capturing not only the cell-to-cell variations, but also to chart their real-time proliferation and the evolution of the same individual cells for long periods.

Here we introduce a novel approach for single-cell studies using the convex lens-induced confinement (CLiC) instrument [7] combined with flow cells containing embedded pits. CLiC is an imaging system that was primarily developed for the isolation and visualization of single molecules in a flow cell made of two glass layers (Figure 1A \& B). The principle of CLiC is as follows: using a lens-rod perpendicular to the focal plane, pressure is applied downward directly onto the flow cell. The resulting deformation of the flow cell confines molecules between the smooth glass surfaces and can load them into features such as pits, which are embedded in the surfaces (Figure 1B) $[8,9]$. This technique had been shown to be able to successfully confine and isolate diffusing [5] and interacting molecules [10-12] for long periods and improve fluorescent signal detection [7]. To date, CLiC has primarily been used to study materials, biomolecules and particles, with only limited information available regarding its suitability for live-cell investigations [13].

In this study, we used the yeast Saccharomyces cerevisiae, which is a well-known and extensively studied model organism used in the investigation of diverse eukaryotic cell biology. The insights gained from yeast are simplified but can be extrapolated into mammalian systems. Due to the high degree of conservation existing between yeast and mammalian genes (over $50 \%$ for essential genes), yeast is often used to understand the mechanisms of action of small molecules and drugs and to acquire preliminary data for drug

\section{The only 2-in-1 pipetter ali- $\mathbf{Q}^{m}$}

\section{EASIER and FASTER}

Repeat Pipetting using Serological Pipets!

Higher Accuracy and Better Repeatability than by Eye!

- Faster and easier multidispensing with NO NEED to watch the lines.

- Repeatable accuracy* with the press of one button!

- Plus aspirate and dispense as you're used to.

- Use any size or brand of serological pipets.

- It's a 2-in-1, regular \& repeating, pipet controller!

Ideal for cell culture, dispensing media, filling plates, tubes, cryovials, etc.

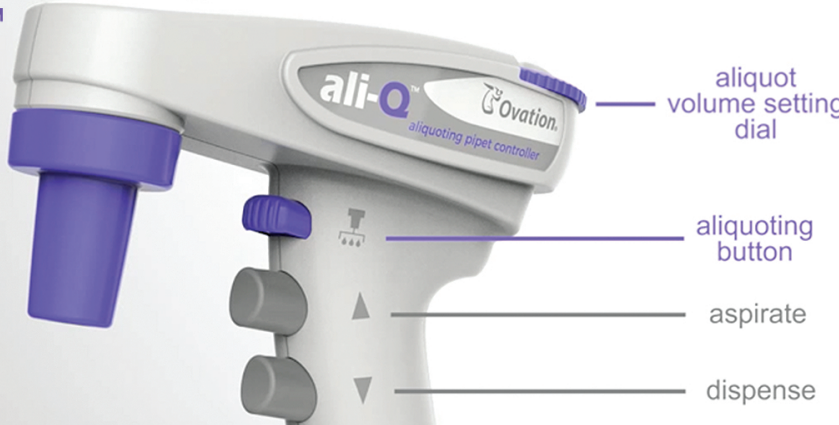

"I love the aliquot feature of this pipette. Especially when I'm transfecting or boosting my cultures, the aliquot saves me a bunch of effort and time."

For more reviews see www.selectscience.net
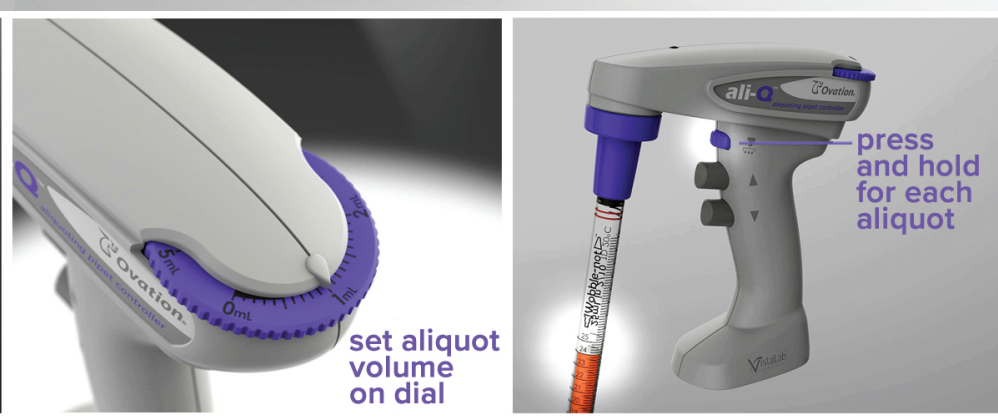
(A)

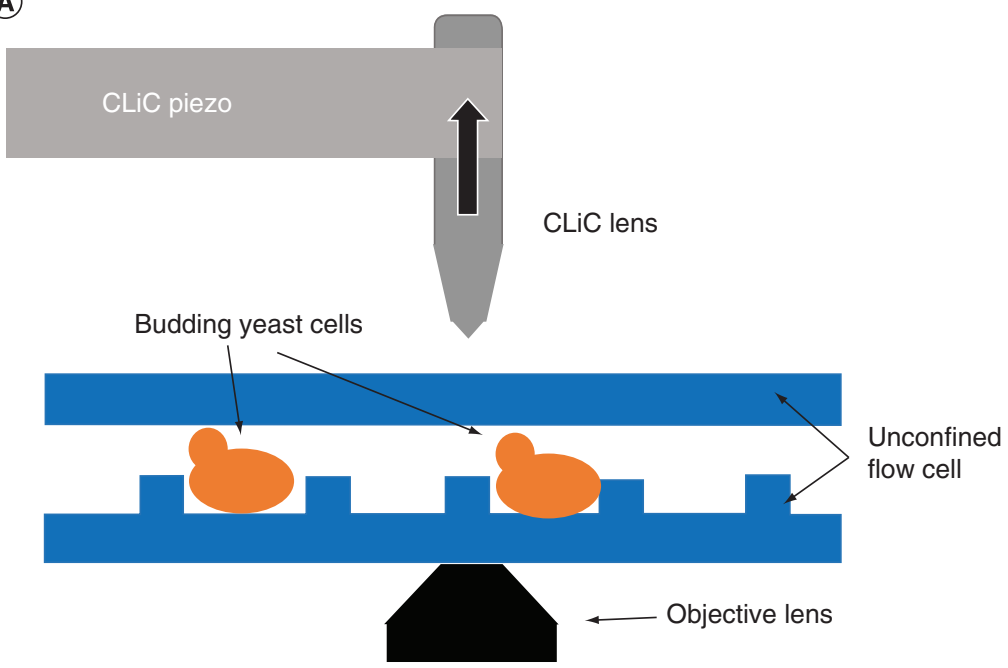

(B)
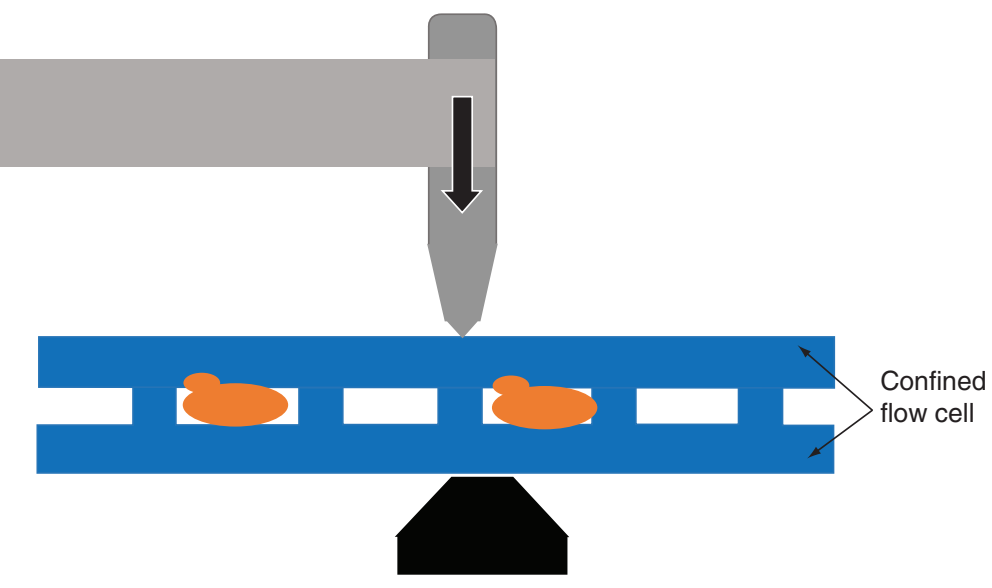

Figure 1. Convex lens-induced confinement instrument. (A) Representative schema of the CLiC instrument with yeast cells loaded into the unconfined flow cell that contains embedded pits.

(B) Schematic representation of the CLiC instrument with the lens rod moving downward to confine the yeast cells into the flow cell pits.

CLiC: Convex lens-induced confinement.

- discovery efforts [14-16]. Additional advantageous criteria make yeast an ideal model organism to benchmark the CLiC cell assay. These criteria include: its low level of genomic instability compared with cultured cells, its simple and low-cost growth requirements, and its short doubling time, which allows faster drug effects and pathway perturbation assessment. We therefore used yeast to validate the $\mathrm{CLiC}$ instrument for single-cell analyses, specifically comparing the CLiC imaging platform to a conventional microscope slide for fluorescent cell imaging, drug response studies and cell-proliferation observations.

\section{MATERIALS \& METHODS}

Yeast cell propagation \& staining

For localization experiments with fluorescent probes, we used BY4743 diploid cells (MATa/

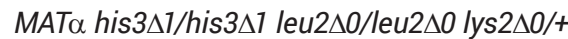
met75 $\Delta 0 /+$ ura3 $\triangle 0 / u r a 3 \Delta 0$ ) generated from a cross between BY4741 and BY4742 [17].

\section{Vacuolar staining}

We inoculated BY4743 cells in $3 \mathrm{ml}$ YPD media and grew them overnight ( $16 \mathrm{~h})$ at $30^{\circ} \mathrm{C}$. We subsequently adjusted the cell suspension to an $\mathrm{OD}_{600 \mathrm{~nm}}$ of 10 , added $4 \mu \mathrm{M}$ FM4-64 vacuolar stain in YPD and incubated on an interval mixer for $30 \mathrm{~min}$ at $30^{\circ} \mathrm{C}$. We then removed the excess probe by centri- fuging cells at $500 \times g$ for $1 \mathrm{~min}$, aspirating the supernatant and washing cells twice in YPD before suspending in $1 \mathrm{ml} \mathrm{YPD}$.

\section{Nuclear DNA live cell staining}

We stained BY4743 cells in mid-log phase with an $\mathrm{OD}_{600 \mathrm{~nm}}$ of $0.5 \mathrm{using} 2.5 \mu \mathrm{g} / \mathrm{ml}$ final concentration of 4',6-diamidino-2-phenylindole (DAPI) on an interval mixer for $30 \mathrm{~min}$ at $30^{\circ} \mathrm{C}$. We then harvested the cell pellet by centrifugation at $500 \times \mathrm{g}$ for $1 \mathrm{~min}$, washed with $1 \times$ phosphate-buffered saline and resuspended in YPD for a final $O_{600 \mathrm{~nm}}$ of approximately 10 .

\section{Mitochondrial staining}

Upon treatment of BY4743 cells with $2 \mathrm{mM}$ methotrexate or $2 \%$ dimethyl sulfoxide (DMSO) as a vehicle control for $20 \mathrm{~h}$, we pelleted and resuspended cells in prewarmed $\left(30^{\circ} \mathrm{C}\right)$ staining solution containing YPD media and $200 \mathrm{nM}$ Mitotracker Red CMXRos. We subsequently incubated the cells at $30^{\circ} \mathrm{C}$ with shaking for $30 \mathrm{~min}$ in a thermomixer, pelleted and resuspended them in fresh YPD media prewarmed at $30^{\circ} \mathrm{C}$.

\section{CONVEX LENS-INDUCED CONFINEMENT}

The CLiC instrument is composed of five major components assembled to allow confinement of single molecules or cells into embedded wells in the CLiC flow cell, which we refer to in this paper as pits. (i) The first component is a mounting stage that perfectly fits into an inverted microscope's main stage. (ii) The second component is a mounting chuck that holds the flow cell, enables fluid exchange and is inserted onto the mounting stage. (iii) The third and main part is the flow cell made of two thin glass coverslips. The bottom coverslip contains embedded wells and is attached to the upper flat coverslip by a $30-\mu \mathrm{m}$ thick double-sided adhesive [11]. Cell samples can be loaded between the two layers through a fluidic access port. (iv) The fourth component is a piezo, which controls the $y$-axis of the lens rod. (v) Last is the lens-rod, which consists of a convex lens, mounted curved-side down, on a narrow rod. When lowered, the lens-rod pushes on the top coverslip, deforming it downward, which consequently restrains cells into the 


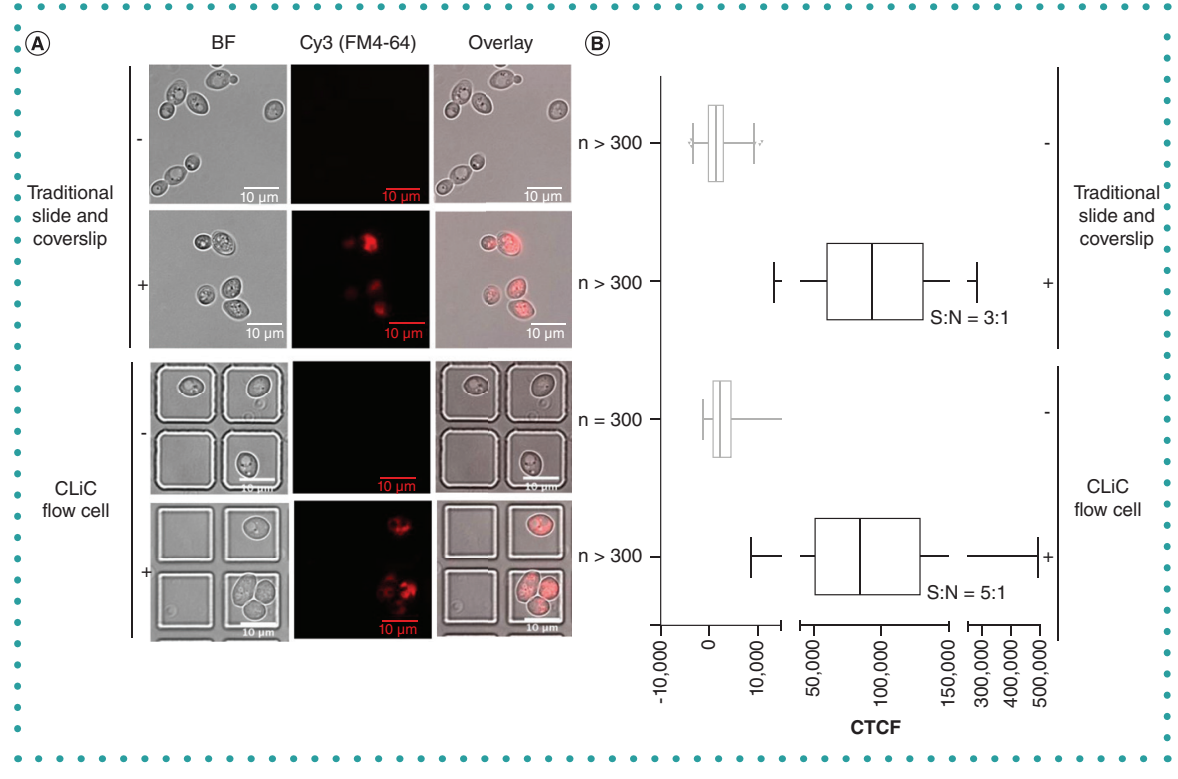

embedded wells. This instrument does not tether or stick down cells, but simply confines them. Cells can then be imaged following the microscope's standard protocols.

\section{WIDE-FIELD MICROSCOPY \\ Image acquisition on microscope slide \& processing}

We used a $63 \times 1.4$ NA oil-immersion objective mounted on a Zeiss (Oberkochen, Germany) Axiovert 200 m microscope, an Excelitas (MA, USA) X-Cite 120 LED light source and a Zeiss AxioCam HRm Rev.2 camera to capture images of cells in brightfield channel exposed for $10 \mathrm{~ms}$ under 4.0-V transmitted light. We used excitation and emission filters appropriate for FM4-64 (Cy3, excitation/emission: 515/640 nm), DAPI (excitation/emission: 359/461 nm) and Mitotracker Red (Cy3, excitation/emission: $579 / 603 \mathrm{~nm}$ ) with $830 \mathrm{~ms}, 125 \mathrm{~ms}$ and $150 \mathrm{~ms}$ of exposure, respectively. Images were captured using the multi-dimensional acquisition setting on the AxioVision 4.8.2 software (Zeiss).

\section{Image acquisition in flow cell \\ \& processing}

For cell imaging using the Scopesys (QC, Canada) CLiC instrument, we loaded $5 \mu$ of the stained cell suspension into the flow cellcontaining embedded pits and imaged with the $63 \times$ oil-immersion objective as described above. We captured images in the brightfield channel using $100 \mathrm{~ms}$ exposure under $11.0 \mathrm{~V}$ transmitted light and in the DAPI or Cy3 fluorescent channels with acquisition settings identical to those used for imaging cells on a microscope slide.

\section{Microscopy}

image analysis \& fluorescent signal quantification

ImageJ software (with the Fiji plugin) was used to measure the 'area', 'integrated intensity' and 'mean gray value' of $\mathrm{fl}$ u orescent cells $[18,19]$. We first selected the regions of interest (ROI) delineating every single cell on channel 1 (brightfield-transmitted channel) and subsequently performed fluorescent signal measurements on channel 2 (fluorescent channel). For normalization, we measured an empty background area on the field of view and determined the corrected total cell fluorescence (CTCF)
Figure 2. Corrected total cell fluorescence of FM4-64 stained cells under confinement in the convex lens-induced confinement instrument flow cell versus on a traditional microscope slide. (A) Representative images of BY4743 unstained (-) or stained with FM4-64 vacuolar stain ( + ) and captured under a traditional microscope slide or when confined in the CLiC flow cell. Imaging performed under BF channel and fluorescent channel (Cy3, FM4-64). (B) Box and Whisker plot of corrected total cell fluorescence distribution for $n \geq 300$ cells per condition. BF: Brightfield; CLiC: Convex lens-induced confinement; CTCF: Corrected total cell fluorescence; S:N: Signal-to-noise ratio.

using the following formula:

$\mathrm{CTCF}=\begin{aligned} & \text { Integrated Density }-(\text { Area of selected cell } \times \\ & \text { Mean fluorescence of background readings })\end{aligned}$

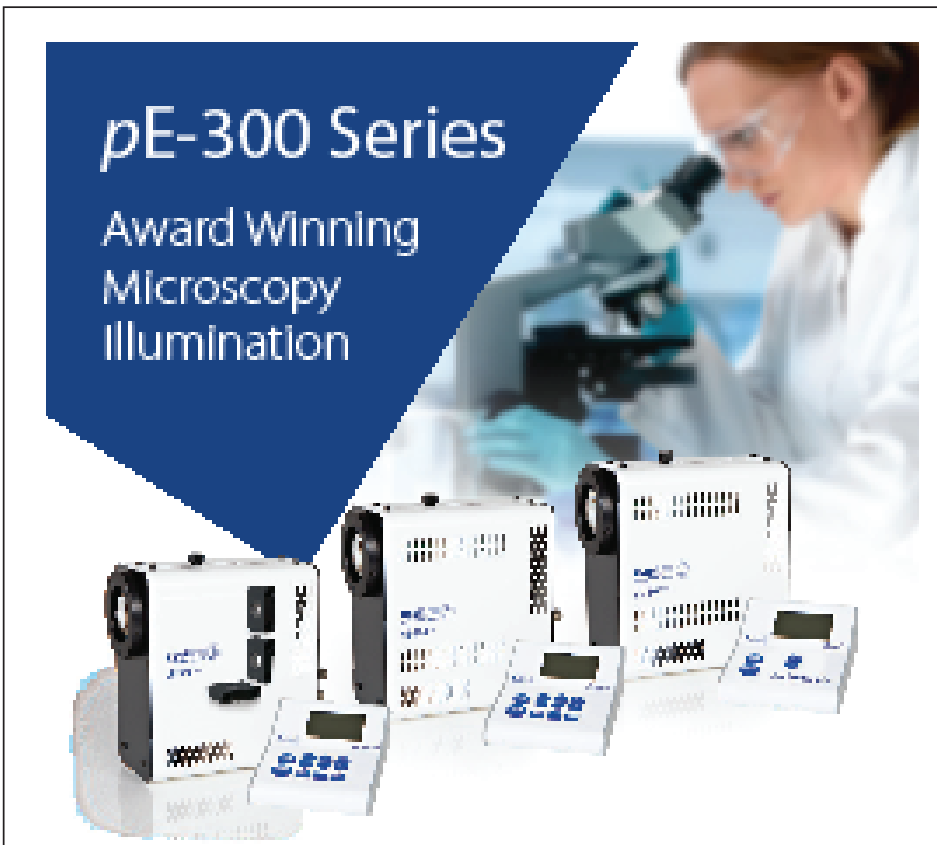

\section{A range of LD Illumination Systers for fucrexcerke, optogerktics electrophysology and hich-speed miruoscopy applications}
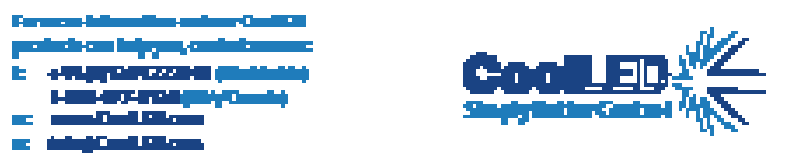
Figure 3. Corrected total cell fluorescence of DAPI-stained cells under confinement in flow cell vs on microscope slide. (A) DAPI-stained BY4743 cells captured under Brightfield (BF) and fluorescent (DAPI) channels. Imaging performed under flow cell confinement (top) and on a microscope slide (bottom). (B) Column scatter plot of CTCF of $n \approx 100$ cells per condition.

Image displays individual data (blue markers), their average (black horizontal lines) and standard deviation (blue error bars) for each condition. $\star \star \star p<0.05$ under an unpaired two-tailed t-test.

BF: Brightfield; CLiC: Convex lensinduced confinement; CTCF: Corrected total cell fluorescence; DAPI: 4',6-diamidino-2-phenylindole.

Figure 4. Mitochondria fluorescent imaging of methotrexate versus dimethyl sulfoxide-treated cells. Mitotrackerstained BY4743 cells treated for $20 \mathrm{~h}$ with $2 \%$ DMSO vehicle control (top) or $2 \mathrm{mM}$ methotrexate (bottom) and captured in flow cells under Brightfield (BF) and Cy3 Fluorescent (Mitotracker Red ${ }^{T M}$ ) channels. BF: Brightfield; DMSO: Dimethyl sulfoxide; MTX: Methotrexate.

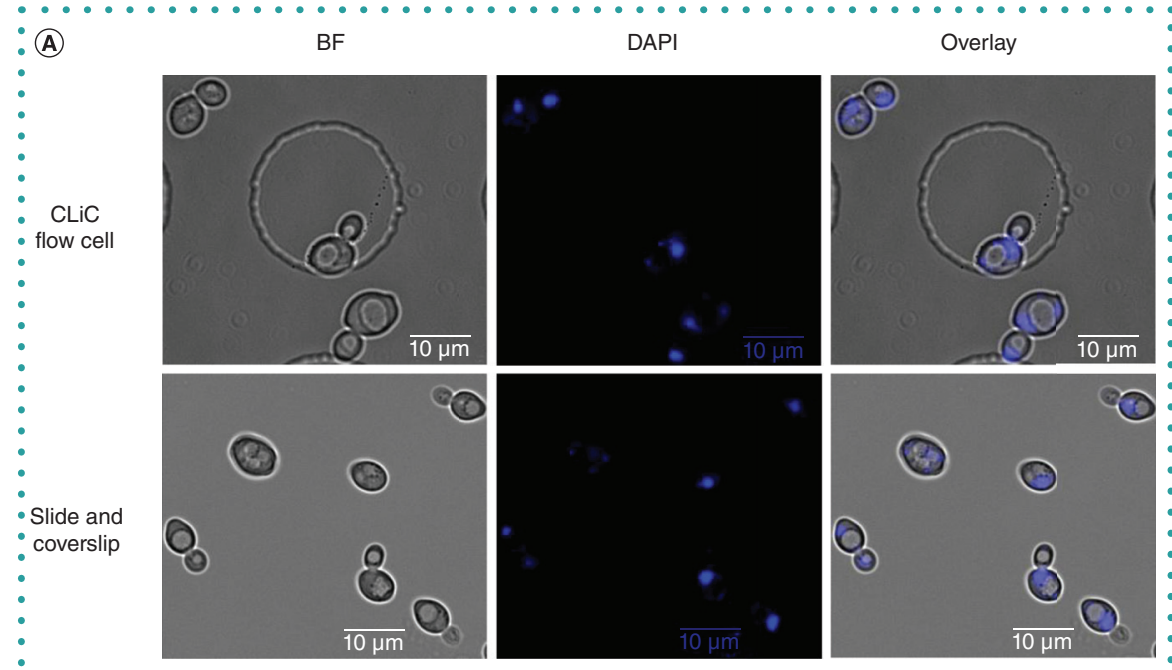

(B)

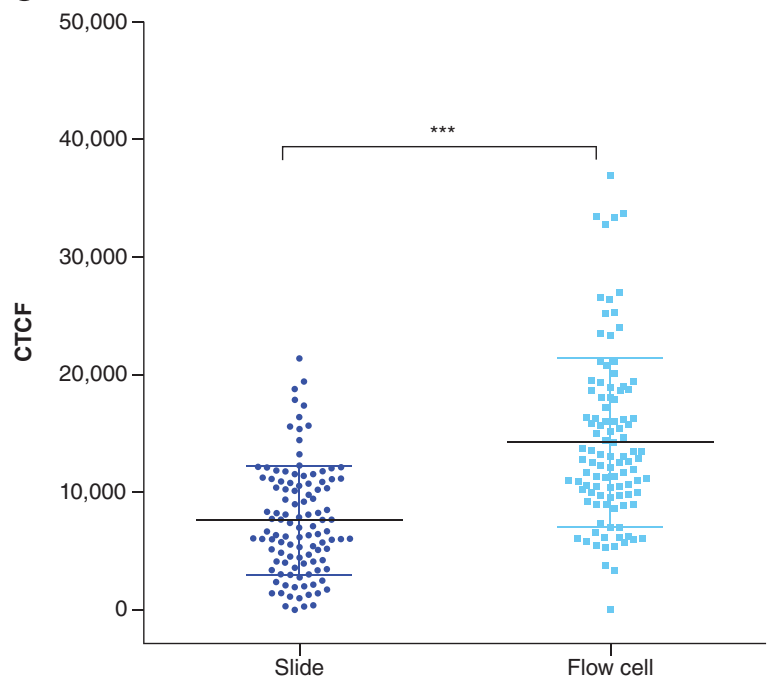

$\mathrm{BF}$
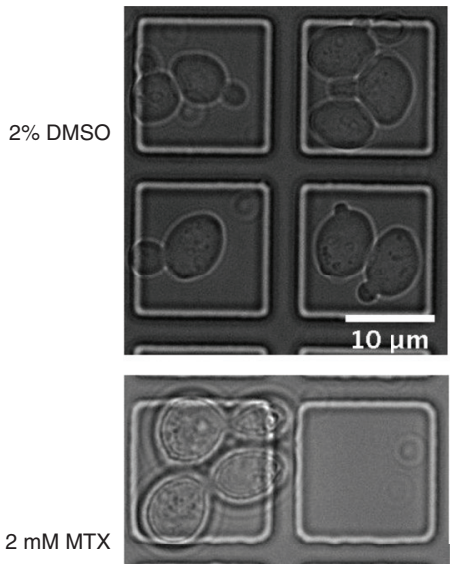

2 mM MTX

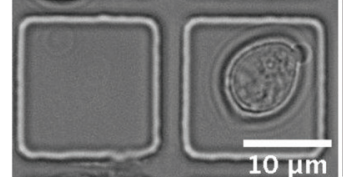

Mitotracker red
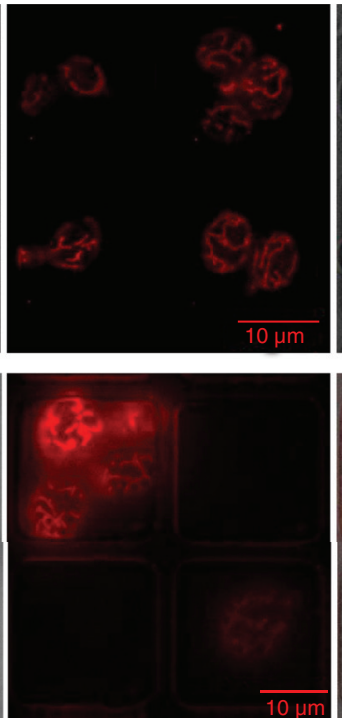

Overlay
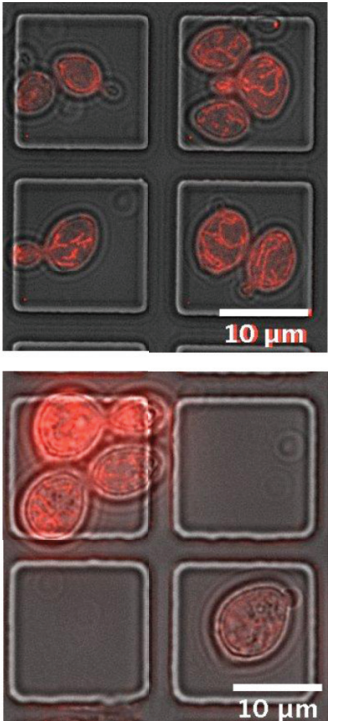


\section{Cell proliferation under}

flask versus flow cell platform

For the growth comparison experiment, we used the $d f r 1 / D F R 1$ heterozygous deletion strain, derived from BY4743 [20]. This strain is deleted for a single copy of the enzyme dihydrofolate reductase, which is the established target of methotrexate [21].

\section{Yeast growth in flasks}

We propagated a cell suspension, characterized by an optical density measured at $600 \mathrm{~nm}\left(\mathrm{OD}_{600 \mathrm{~nm}}\right)$ of 0.25 , in $50 \mathrm{ml}$ YPD in a flask at $30^{\circ} \mathrm{C}$ for three generations, and performed readings with an Eppendorf (Hamburg, Germany) Biophotometer every $15 \mathrm{~min}$.

\section{Yeast growth in flow cell}

We loaded $5 \mu$ l of a cell suspension with an $\mathrm{OD}_{600 \mathrm{~nm}}$ of 5 into a flow cell, and grew the cells at $30^{\circ} \mathrm{C}$. To monitor and control the temperature of the flow cell environment, we used a silicon heat tracing cable and temperature sensor wrapped around the 63x oil-immersion objective. The Zeiss Axiovert $200 \mathrm{~m}$ microscope captured images of proliferating cells every $15 \mathrm{~min}$ for $8 \mathrm{~h}$.

\section{Viability assay}

To prepare the samples for the spot assay, we grew BY4743 cells in the presence of 2.0, $1.0,0.5$ or $0.25 \mathrm{mM}$ methotrexate, or $2 \%$ DMSO vehicle control for $20 \mathrm{~h}$, normalized them to an $\mathrm{OD}_{600 \mathrm{~nm}}$ of 1 and transferred them to a 96-well plate. We subsequently performed fivefold serial dilutions on each sample, spotted them onto YPD agar by transferring $5 \mu$ of each suspension, and grew the cells at $30^{\circ} \mathrm{C}$ for $48 \mathrm{~h}$ prior to image acquisition.

\section{RESULTS \& DISCUSSION \\ Fluorescent signal detection comparison in flow cell versus microscope slide}

To study the ability to obtain fluorescent signals from live cells on the CLiC instrument and compare the data to that obtained from conventional microscope slides, we captured images of BY4743 cells stained with $4-\mu \mathrm{M}$ FM4-64 vacuolar rim stain on a microscope slide and a CLiC flow cell under similar fluorescent settings. An unstained BY4743 negative control sample was also analyzed. Both platforms detected fluorescent signals consistent with vacuolar rims in stained cells

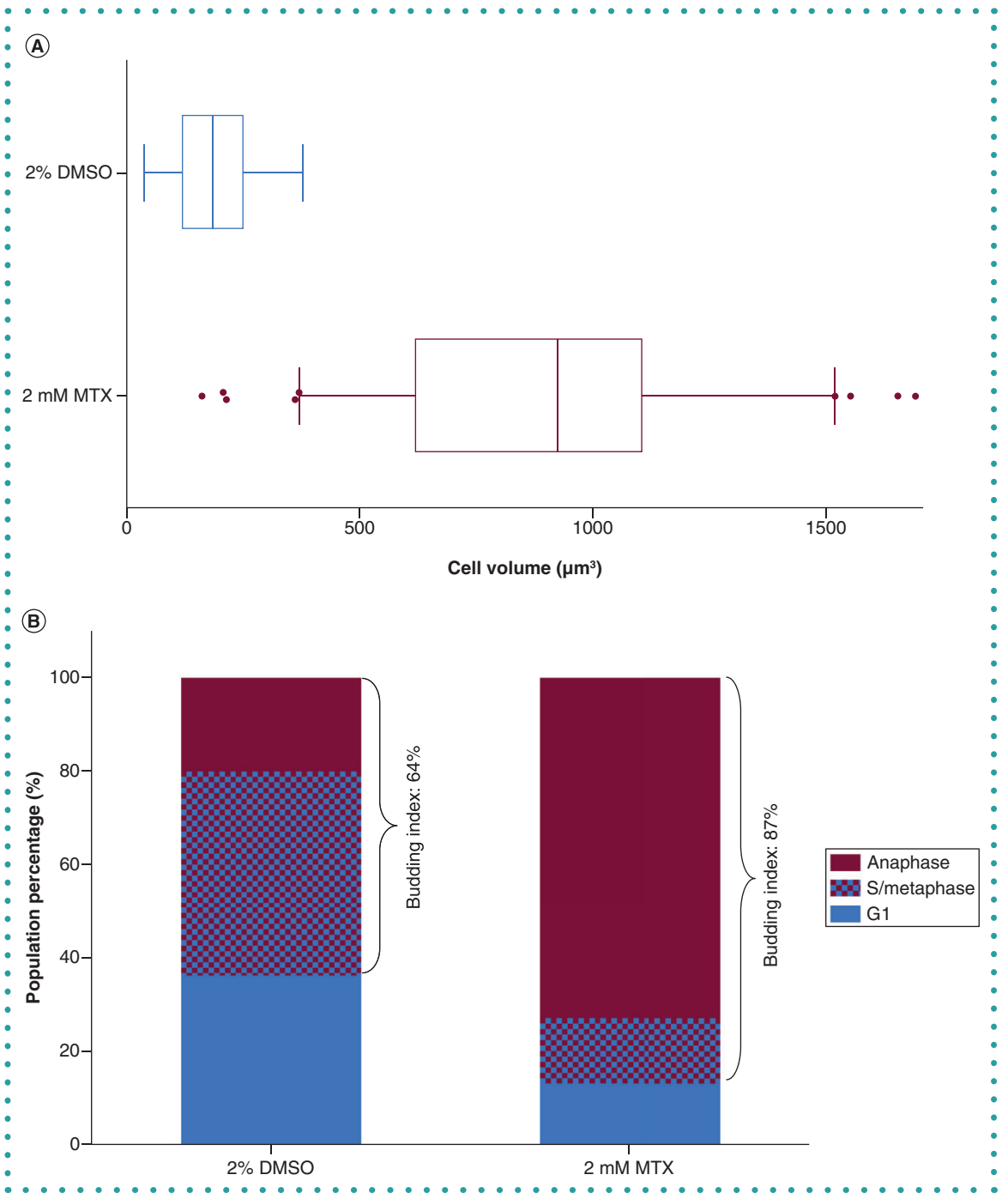

Figure 5. Cell size comparison of methotrexate- versus $2 \%$ dimethyl sulfoxide-treated cells. (A) Box and Whisker plot representation of the cell volume distribution of $n=100$ BY4743 cells treated for $20 \mathrm{~h}$ with $2 \mathrm{mM}$ MTX versus the control treated with $2 \%$ DMSO. (B) Population percentage of cells in anaphase, S/metaphase and G1 phase of the cell cycle and the deduced budding indices of MTXversus DMSO-treated cells.

DMSO: Dimethyl sulfoxide; MTX: Methotrexate.

(Figure 2A). From visual inspection, the CLiC instrument captured the stained vacuolar rims with high contrast. Quantitatively, the average signal-to-noise ratio with CLiC was enhanced compared with the microscope slide, 5:1 versus 3:1, respectively (Figure 2B). The CTCF distribution of the stained samples on the two platforms were not significantly different; however, the distribution obtained with CLiC extended over a larger dynamic range of signals, highlighting the $\mathrm{CLiC}$ ability to detect higher signals (Figure 2B).

Signal detection of cells stained with $2.5 \mu \mathrm{g} / \mathrm{ml}$ DAPI for $30 \mathrm{~min}$ also demonstrated signal detection sensitivity differences between the CLiC flow cell versus the microscope slide. The average integrated fluorescence per stained cell was 10,517 for $n=110$ cells visualized on a microscope slide compared with 17,192 for $n=104$ cells imaged under confinement with CLiC. Under a student's two-tailed t-test, assuming equal variances between the samples, the distribution of signals was significantly higher in CLiC flow cells $(p<0.05)$ (Figure 3A \& B).

The fluorescence signal detection comparison validated the ability of the CLiC instrument to image cellular compartments with high resolution and precision (Figures 2A \& 3A). Additionally, DAPI fluorescent signal detection was greater with increased sensitivity in CLiC flow 


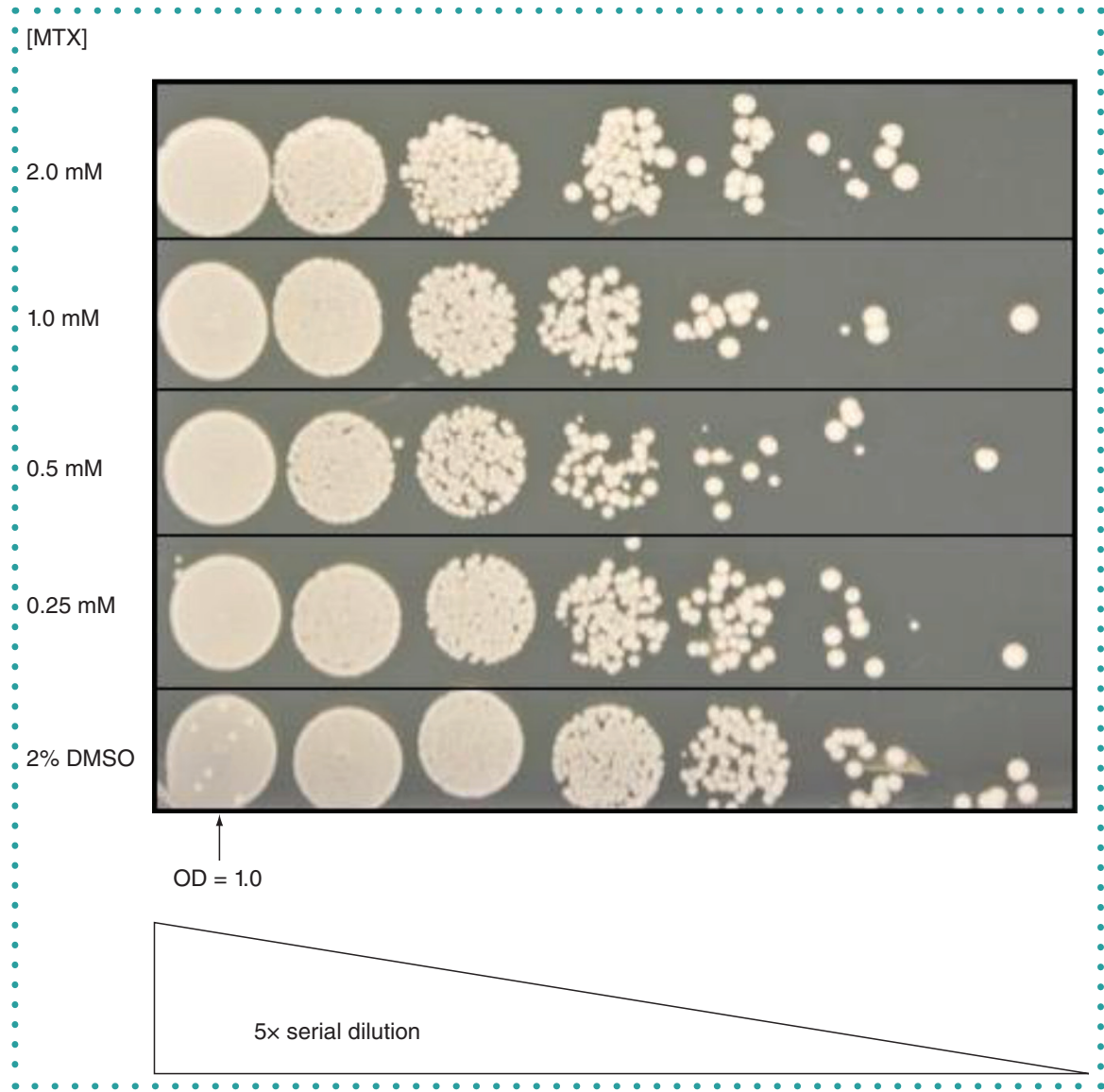

Figure 6. Viability assay on methotrexate-treated BY4743 cells. Cells treated for $20 \mathrm{~h}$ with $2.0 \mathrm{mM}$, $1.0,0.5$ or $0.25 \mathrm{mM} \mathrm{MTX}$, or $2 \%$ DMSO, spotted onto YPD agar and incubated for $48 \mathrm{~h}$ are displayed on this image. From left to right, spots correspond to $5 \times$ serially diluted cells starting with a cell suspension with an $\mathrm{OD}_{600 \mathrm{~nm}}$ of 1.0 .

DMSO: Dimethyl sulfoxide; MTX: Methotrexate.

$\checkmark$ cells compared with microscope slides. This higher sensitivity could be explained by the decrease in the vertical volume of the cells when squeezed, allowing higher background signal rejection and better fluorophore detection [8].

\section{Methotrexate effect}

on yeast in flow cells

After validating that the $\mathrm{CLiC}$ instrument was suitable for fluorescence imaging, this platform was used to study the effect of the antifolate drug methotrexate, a dihydrofolate reductase inhibitor, on BY4743 cells [21]. In yeast, the dihydrofolate reductase enzyme is encoded by DFR1 and is required for tetrahydrofolate biosynthesis. This enzyme is functionally conserved between yeast and human cells; that is, the viability and antifolate resistance of a yeast $d f r 7$ mutant can be rescued by expression of the human enzyme DHFR [20]. Inhibition by metho- trexate results in fitness defects of yeast cells, which is likely due to the adverse effects of the drug on nucleic acid and amino acid biosynthesis [21]. Dfrr has previously been reported to be necessary for mitochondrial morphology maintenance in the presence of $10 \mu \mathrm{g} / \mathrm{ml}$ doxycycline [22]. We therefore hypothesized that inhibiting Dfr1 with methotrexate would have an impact on the mitochondrial structure of the cells.

BY4743 cells treated with $2.0 \mathrm{mM}$ methotrexate for $20 \mathrm{~h}$ showed no major mitochondrial structural damage based on observations of Mitotracker Red stained cells (Figure 4). Methotrexate treatment did, however, result in a drastic morphology change in BY4743 cells. Treated cells were almost fivefold larger in volume than the $2 \%$ DMSO-treated cells, with an average volume \pm SEM of $900.8 \pm 35.41 \mu \mathrm{m}^{3}(n=100)$ compared with $185.9 \pm 8.168 \mu \mathrm{m}^{3}(n=100)$, respectively. Most strikingly, methotrexate had a pronounced effect on cell cycle progression. When assayed for budding index [23], we found a 1.36-fold increase in the population budding index of $\mathrm{n} \approx$ 200 treated cells $(87 \%)$ compared with the DMSO-treated control (64\%) (Figure 5A \& B). When treated with methotrexate, $73 \%$ of the cell population was found in the telophase/ anaphase stage of the cell cycle compared with $16 \%$ for the DMSO-treated control, suggesting that methotrexate either caused mitotic arrest or delayed mitotic exit [24].

To further investigate methotrexate's effect on BY4743 cell viability, cells treated with $2.0 \mathrm{mM}$ methotrexate for $20 \mathrm{~h}$ were spotted onto YPD agar for $48 \mathrm{~h}$. To our surprise, despite their dramatic morphology change, budding alterations and growth inhibition (Figures 3, 4A \& 4B), methotrexate treatment did not exhibit a major impact on the cell viability following recovery on YPD in the absence of methotrexate (Figure 6). This observation suggests that methotrexate is cytostatic and not cytocidal under the conditions tested.

\section{Yeast cell growth study in flow cell}

To investigate whether the $\mathrm{CLiC}$ instrument is useful for observing live cell growth, cells in log phase were re-suspended in YPD, adjusted to an $\mathrm{OD}_{600 \mathrm{~nm}}$ of 5 and loaded into a flow cell confined by CLiC. Observations recorded at $30^{\circ} \mathrm{C}$ using brightfield illumination (refer to 'Materials \& methods' for temperature control). Confined single cells had an initial doubling time that ranged from $1 \mathrm{~h} 30 \mathrm{~min}$ to $2 \mathrm{~h} 30 \mathrm{~min}$ (Figure 7A \& B), with an average of $2 \mathrm{~h} 15 \mathrm{~min}$, which was consistent with the first doubling time recorded when the same strain is grown in flasks (Figure 7C \& D, Supplementary Video 1). With the CLiC instrument and a cell suspension with an initial $\mathrm{OD}_{600 \mathrm{~nm}}$ of 5 prior to loading, we were able to reproducibly observe two cell doublings (Supplementary Videos 1-4). This assay validated the use of the CLiC instrument for single cell proliferation studies and could be used to observe the real-time drug response variabilities among a cell population.

This study validated the use of the CLiC instrument for accurate single-cell investigations. We observed that fluorescently labelled cells had improved background rejection (i.e., lower nonspecific fluorescence), which resulted in a higher signal-to-noise ratio within the flow cell compared with the microscope 
(A)



(C)

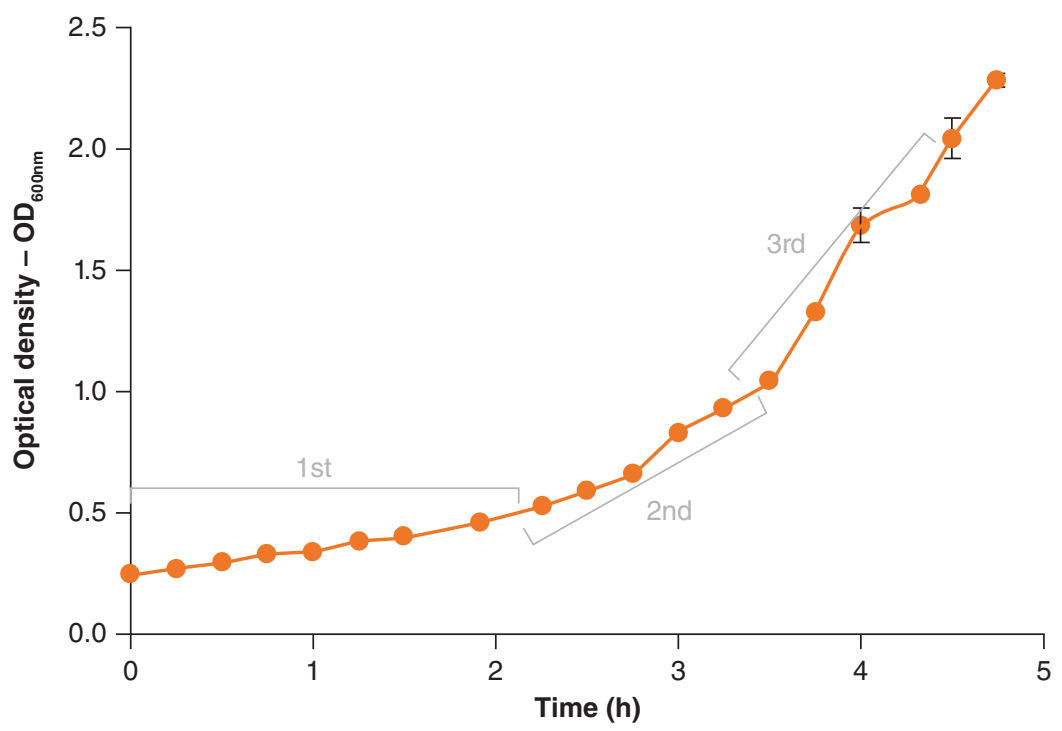

(B)

\begin{tabular}{|c|c|}
\hline $\begin{array}{l}\text { Cells in flow } \\
\text { cell pit \# }\end{array}$ & $\begin{array}{l}\text { Average } \\
\text { doubling time }\end{array}$ \\
\hline 1 & $1 \mathrm{~h} 30 \mathrm{~min}$ \\
\hline 2 & $2 \mathrm{~h} 15 \mathrm{~min}$ \\
\hline 3 & $2 \mathrm{~h} 30 \mathrm{~min}$ \\
\hline 4 & $1 \mathrm{~h} 45 \mathrm{~min}$ \\
\hline 5 & $1 \mathrm{~h} 30 \mathrm{~min}$ \\
\hline 6 & $2 \mathrm{~h} 00 \mathrm{~min}$ \\
\hline 7 & $2 \mathrm{~h} 15 \mathrm{~min}$ \\
\hline 8 & $2 \mathrm{~h} 15 \mathrm{~min}$ \\
\hline 9 & $1 \mathrm{~h} 45 \mathrm{~min}$ \\
\hline 10 & $1 \mathrm{~h} 45 \mathrm{~min}$ \\
\hline 11 & $1 \mathrm{~h} 45 \mathrm{~min}$ \\
\hline 12 & $1 \mathrm{~h} 45$ min \\
\hline 13 & $1 \mathrm{~h} 30 \mathrm{~min}$ \\
\hline 14 & $1 \mathrm{~h} 30 \mathrm{~min}$ \\
\hline 15 & $2 \mathrm{~h} 15 \mathrm{~min}$ \\
\hline 16 & $1 \mathrm{~h} 45 \mathrm{~min}$ \\
\hline 17 & $1 \mathrm{~h} 45 \mathrm{~min}$ \\
\hline 18 & $1 \mathrm{~h} 45 \mathrm{~min}$ \\
\hline 19 & $1 \mathrm{~h} 30 \mathrm{~min}$ \\
\hline Average & $2 \mathrm{~h} 15 \mathrm{~min}$ \\
\hline
\end{tabular}

(D)

\begin{tabular}{|l|l|}
\hline \multicolumn{2}{|c|}{ Cell growth in flask } \\
\hline Doubling time & Time (h) \\
\hline $1 \mathrm{st}$ & $\simeq 2 \mathrm{~h} 10 \mathrm{~min}$ \\
\hline $2 \mathrm{nd}$ & $\simeq 1 \mathrm{~h} 18 \mathrm{~min}$ \\
\hline $3 \mathrm{rd}$ & $\simeq 1 \mathrm{~h} 05 \mathrm{~min}$ \\
\hline
\end{tabular}

Figure 7. Yeast cell growth comparison in convex lens-induced confinement instrument flow cell versus flask. (A) Timepoint 00:00 image of cells confined in convex lens-induced confinement flow cell and grown at $30^{\circ} \mathrm{C}$ in YPD for $8 \mathrm{~h}$ (refer to Supplementary Video 1 for complete growth cycle) and numeric labeling of pits containing cells. (B) Average doubling time of cells in Figure 7A numerically labelled pits. (C) Growth curve of cells grown in flask at $30^{\circ} \mathrm{C}$ with shaking in $Y P D, n=2$. (D) First, second and third doubling time of cells grown in flasks and deduced from Figure $7 \mathrm{C}$.

slides. The platform detected cellular DNA with higher precision and sensitivity, which also applied to other cellular compartments (vacuoles and mitochondria in this study). Most importantly, the CLiC instrument allows robust growth of yeast cells during single-cell confinement and observation in real time. This device could be used in future studies to observe the real-time emergence of resistant drug suppressors as well as yeast and bacterial persistence [4], a phenomenon whereby, upon drug treatment, a small surviving subgroup regrows into a population that has not acquired any resistance and is still as sensitive to the treatment. This device also holds great potential for routine use in fields such as cancer research, where single-cell variabilities and instabilities are especially important for developing effective therapies. While we focused on yeast cells as a model system in this work, previous studies performed on adherent Chinese hamster ovary ( $\mathrm{CHO}-\mathrm{K} 1)$ cells demonstrated the feasibility of customizing the $\mathrm{CLiC}$ instrument with a cytoindentor, which allows precise indentation and imaging of live mammalian cells without significantly changing their oxidative stress or causing detectable impacts on cell fitness [13]. To manipulate cell adherence, the flow cells could be customized and coated with fibronectin as previously demonstrated [13]. Further optimization and validation studies will, however, be 
- needed for application on drug response and proliferation studies of mammalian cell lines. As with any single-cell technique, we acknowledge that drug response analysis on mammalian cells might be challenging with the CLiC instrument and not completely reflect their response in vivo. Nevertheless, singlecell technologies are essential to understand cell-to-cell variations in a heterogeneous population. Additionally, with the open architecture of the flow cell, it is possible to rapidly modify the environment for growth optimization (e.g., a stage-top incubator system enclosing the CLiC instrument can be used to maintain the temperature and humidified $\mathrm{CO}_{2}$ for mammalian cells). Based on the data collected during this investigation, we suggest that CLiC is a useful new tool for single-cell biology and is well-suited for the study of single-cell heterogeneity in drug response.

\section{FUTURE PERSPECTIVE}

While extraordinary progress has been made in developing and implementing highthroughput, high-content screening platforms with powerful analysis software, there are still obstacles to wide adoption of real-time cellular imaging. These challenges can be broadly grouped into: hardware, data collection and analysis, and cost and ease of use. With regards to the hardware issue, the current imaging platforms can be categorized as: i) traditional microscopes that have been automated; ii) automated, dedicated imagers; iii) plate readers with single-cell resolution; and iv) flow cytometers. Each of these platforms is capable of single-cell resolution, but some are unable to re-identify and track individual cells over time (e.g., plate readers and cytometers), and even dedicated imagers struggle to faithfully track large numbers of individual cell trajectories.

The flow cell and CLiC instrument therefore fills several important gaps; it is a relatively inexpensive accessory that can be added to any inverted microscope and, most importantly, the confinement afforded by CLic, combined with flow cells containing ordered features, allows one to track individual cell trajectories over an extended period in real-time in physiological conditions. This capability is key to understanding the individual cell's response to drugs or other perturbations. This is especially true when performing highresolution phenotyping. The improved signal that can be obtained from fluorescently labeled molecules should aid in the subcellular localization of ligands in single cells.

While generating arrays of cells within an imaging field is not a new idea [25], Clic provides a flexible (the only constraints are in the initial flow cell design) means to achieve living cell microarrays that are unique in that no tethers are required to keep cells within the focal plane over extended periods. The next challenges with $\mathrm{CLiC}$ as applied to phenotypic screens will be to increase the capacity of the flow cells and to introduce automation, for example, for loading flows cells and liquid exchanges.

\section{SUPPLEMENTARY DATA}

To view the supplementary data that accompany this paper please visit the journal website at: www.future-science. com/doi/suppl/10.2144/btn-2019-0067

\section{AUTHOR CONTRIBUTIONS}

NKT, NC, DB, SL and CN designed and supported the microscopy experiments; NKT acquired and analyzed the data; RT developed and fabricated the flow cells. NKT, $\mathrm{SL}$ and $\mathrm{CN}$ wrote the manuscript.

\section{ACKNOWLEDGMENTS}

We thank ScopeSys (www.scopesys.ca) for access to the CLiC technology package. The project was supported by a grant from the $\mathrm{CFI}$ to $\mathrm{CN}$.

\section{FINANCIAL \& COMPETING INTERESTS DISCLOSURE}

SL acknowledges the NSERC Discovery, Accelerator, and Idea to Innovation programs as well as McGill University for research funding and support. SL and DB have a financial interest in ScopeSys, the company which is commercializing $\mathrm{CLiC}$ imaging. The authors have no other relevant affiliations or financial involvement with any organization or entity with a financial interest in or financial conflict with the subject matter or materials discussed in the manuscript apart from those disclosed.

No writing assistance was utilized in the production of this manuscript.

\section{OPEN ACCESS}

This work is licensed under the AttributionNonCommercial-NoDerivatives 4.0 Unported License. To view a copy of this license, visit http://creativecommons.org/licenses/ by-nc-nd/4.0/

\section{REFERENCES}

1. Abdallah BY, Horne SD, Stevens JB et al. Single cell heterogeneity. why unstable genomes are incompatible with ogeneity. why unstable genomes are incompatible with
average profiles. Cell Cycle 12(23), 3640-3649 (2013).

2. Heng HH, Bremer SW, Stevens JB et al. Chromosomal instability (CIN): what it is and why it is crucial to cancer evolution. Cancer Metastasis Rev. 32(3-4), 325-340 (2013).

3. Elowitz MB, Levine AJ, Siggia ED, Swain PS. Stochastic gene expression in a single cell. Science 297(5584), 1183-1186 (2002).

4. Balaban NQ, Merrin J, Chait R, Kowalik L, Leibler S. Bacterial persistence as a phenotypic switch. Science 305(5690), 1622-1625 (2004)

5. Baltekin Ö, Boucharin A, Tano E, Andersson DI, Elf J. Antibiotic susceptibility testing in less than $\mathbf{3 0}$ min using direct single-cell imaging. Proc. Natl Acad. Sci. USA 114(34) 9170-9175 (2017).

6. Dai J, Hamon M, Jambovane S. Microfluidics for antibiotic susceptibility and toxicity testing. Bioengineering (Basel) 3(4), E25 (2016).

7. Leslie SR, Fields AP, Cohen AE. Convex lens-induced confinement for imaging single molecules. Anal. Chem. 82(14), 6224-6229 (2010).

8. Berard D, McFaul CM, Leith JS, Arsenault AK, Michaud F, Leslie SR. Precision platform for convex lens-induced confinement microscopy. Rev. Sci. Instrum. 84(10), 103704 (2013).

9. Berard DJ, Shayegan M, Michaud F, Henkin G, Scott S, Leslie $S$. Formatting and ligating biopolymers using adjustable nanoconfinement. Appl. Physics Lett. 109(3), 033702 (2016).

10. Scott $S, X u Z Z M$, Kouzine $F$ et al. Visualizing structuremediated interactions in supercoiled DNA molecules. Nucleic Acids Res. 46(9), 4622-4631 (2018).

11. Scott S, Shaheen C, McGuinness B et al. Single-molecule visualization of the effects of ionic strength and crowding on structure-mediated interactions in supercoiled DNA molecules. Nucleic Acids Res. 47(12), 6360-6368 (2019).

12. Shayegan $M$, Tahvildari R, Metera K, Kisley L, Michnick SW, Leslie SR. probing inhomogeneous diffusion in the microenvironments of phase-separated polymers under confinement. J. Am. Chem. Soc. 141(19), 7751-7757 (2019).

13. Jia B, Wee TL, Boudreau CG et al. Parallelized cytoindentation using convex micropatterned surfaces. BioTechniques 61(2), 73-82 (2016).

14. Simon JA, Bedalov A. Yeast as a model system for anticancer drug discovery. Nat. Rev. Cancer 4(6), 481-492 (2004)

15. Giaever $G$, Nislow $C$. The yeast deletion collection: a decade of functional genomics. Genetics 197(2), 451-465 (2014).

16. Lee AY, St Onge RP, Proctor MJ et al. Mapping the cellular response to small molecules using chemogenomic fitness signatures. Science 344(6180), 208-211 (2014).

17. Brachmann CB, Davies A, Cost GJ et al. Designer deletion strains derived from Saccharomyces cerevisiae S288C: a strains derived from Sacharomyces cerevisiae S288C: a disruption and other applications. Yeast 14(2), 115-132 (1998).

18. Schneider CA, Rasband WS, Eliceiri KW. NIH Image to ImageJ: 25 years of image analysis. Nat. Methods 9(7), 671-675 (2012).

19. Schindelin J, Arganda-Carreras I, Frise E et al. Fiji: an opensource platform for biological-image analysis. Nat. Methods 9(7), 676-682 (2012).

20. Wong LH, Sinha S, Bergeron JR et al. Reverse chemical genetics: comprehensive fitness profiling reveals the spectrum of drug target interactions. PLoS Genet. 12(9), e1006275 (2016).

21. Lagosky PA, Taylor GR, Haynes RH. Molecular characterization of the Saccharomyces cerevisiae dihydrofolate reductase gene (DFR1). Nucleic Acids Res. 15(24), 10355-10371 (1987).

22. Altmann $\mathrm{K}$, Westermann $\mathrm{B}$. Role of essential genes in mitochondrial morphogenesis in Saccharomyces cerevisiae. Mol. Biol. Cell 16(11), 5410-5417 (2005).

23. Zettel MF, Garza LR, Cass AM et al. The budding index of Saccharomyces cerevisiae deletion strains identifies genes important for cell cycle progression. FEMS Microbiol. Lett. 223(2), 253-258 (2003).

24. Bi E, Park $\mathrm{HO}$. Cell polarization and cytokinesis in budding yeast. Genetics 191(2), 347-387 (2012).

25. Jonczyk R, Kurth T, Lavrentieva A, Walter JG, Scheper T, Stahl F. Living cell microarrays: an overview of concepts. Microarrays (Basel) 5(2), (2016). 